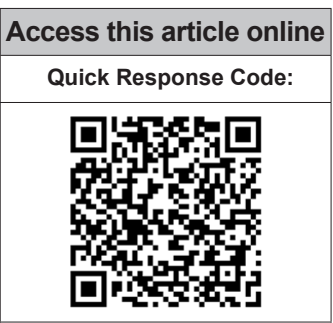

Website:

www.jponline.org

DOI:

10.4103/JLP.JLP_173_18
Departments of Microbiology and ${ }^{1}$ Critical Care Medicine, S. L. Raheja Hospital, Mumbai, Maharashtra, India

Address for correspondence:

Dr. Dhruv Mamtora,

Department of

Microbiology,

S. L. Raheja Hospital,

Raheja Rugnalaya Marg, Mahim West, Mahim,

Mumbai - 400 016,

Maharashtra, India

E-mail: dhruv_mamtora@ yahoo.com

Submission: 29-12-2018 Accepted: 24-02-2019

\title{
Microbiological profile and antibiotic susceptibility pattern of Gram-positive isolates at a tertiary care hospital
}

\author{
Dhruv Mamtora, Sanjith Saseedharan', Pallavi Bhalekar, Surekha Katakdhond
}

\section{Abstract:}

OBJECTIVES: Gram-positive infections such as those by Staphylococcus aureus have contributed to the disease burden by increasing the morbidity and mortality rates in India. This study aims to determine the prevalence and the antibiotic susceptibility pattern of Gram-positive pathogens at a tertiary care hospital, Mumbai, Maharashtra, India.

MATERIALS AND METHODS: This retrospective cross-sectional study was carried out from January, 2015 to December, 2017, at a tertiary care hospital in Mumbai, India. The clinical isolates were cultured, and identification was done using Vitek 2 culture system. The antibiotic susceptibility testing was done as per the Clinical Laboratory Standard Institute guidelines.

RESULTS: Out of $2132(29 \%)$ Gram-positive isolates, S. aureus (49\%) was the most common encountered pathogen, followed by Enterococcus spp. (24.5\%) and coagulase-negative Staphylococcus (16\%). Majority of the S. aureus were observed in patients with skin and soft-tissue infections $(61.2 \%)$ followed by those suffering from respiratory $(41 \%)$ and bloodstream infections $(35 \%)$. Among the infections caused by $S$. aureus, the prevalence of methicillin resistance was $30 \%$. While the MRSA isolates showed lower sensitivity toward co-trimoxazole (39\%), clindamycin $(30 \%)$, erythromycin (23\%), and ciprofloxacin (10\%), they showed higher susceptibility to linezolid (98\%), vancomycin $(98 \%)$, and teicoplanin $(98 \%)$. All the isolates were found to be sensitive to daptomycin and tigecycline. While vancomycin-resistant enterococci (VRE) formed $7.5 \%$, the linezolid-resistant enterococcus species was as high as $4.1 \%$.

CONCLUSION: The study showed a high prevalence of MRSA and VRE, thereby emphasizing the increasing antimicrobial resistance pattern of the Gram-positive pathogens. Therefore, there is an urgent need for novel antimicrobial stewardship to restrict the ongoing resistance rate among the isolates.

Key words:

Antibiotic susceptibility pattern, Gram-positive pathogen, methicillin-resistant Staphylococcus aureus, prevalence, vancomycin-resistant enterococci

\section{Introduction}

The 1918 "Spanish flu" is one of the few I examples of an infectious disease that has plagued humans and has prominently marked itself in history. More than a century now, infectious diseases are still the leading causes of disability, morbidity, and mortality worldwide. ${ }^{[1,2]}$ In India

This is an open access journal, and articles are distributed under the terms of the Creative Commons Attribution-NonCommercial-ShareAlike 4.0 License, which allows others to remix, tweak, and build upon the work non-commercially, as long as appropriate credit is given and the new creations are licensed under the identical terms.

For reprints contact: reprints@medknow.com too, high rate of infections is a public health threat which is not only associated with increased mortality rate, but also responsible for disability and prolonged hospital stay. ${ }^{[3]}$ The crude mortality rate in India is $417 / 100,000$ persons..$^{[4]}$ Over the years, the poor antimicrobial stewardship in India has led to an increase in multidrug-resistant (MDR) pathogens in both community and hospital settings. ${ }^{[4,5]}$

How to cite this article: Mamtora D, Saseedharan S, Bhalekar P, Katakdhond S. Microbiological profile and antibiotic susceptibility pattern of Gram-positive isolates at a tertiary care hospital. J Lab Physicians 2019;11:144-8. 
Gram-positive pathogens have played a crucial role in common infections such as sepsis, pneumonia, and urinary tract infections. Staphylococcus aureus is the major Gram-positive pathogen related to clinical infections ranging from bacteremia, skin and soft-tissue infections to device-related infections. ${ }^{[6]}$ Methicillin Resistant $S$. aureus (MRSA) is a life-threatening nosocomial pathogen whose resistance pattern is contributed by either multiple mutations or by horizontal gene transfer. Moreover, according to national guidelines on antimicrobial use, patients infected with MRSA have a high mortality rate ${ }^{[7]}$ which could be due to various factors such as late detection, manifestation of the underlying susceptibility to infections, and past antibiotic overuse. A recent multicentric study has reported the overall MRSA prevalence to be $37.3 \% ;{ }^{[8]}$ however, other studies have reported the prevalence to range from $20.2 \%$ to $80.4 \%$ depending on the geographical region. ${ }^{[9-12]}$

Apart from MRSA, another potential threat is the emergence of MDR enterococci, especially vancomycin-resistant enterococci (VRE). Enterococcus species consisting of Enterococcus faecalis and Enterococcus faecium are the most common causative pathogens of urinary tract infections followed by bloodstream infections (BSIs). ${ }^{[13]}$ Indian studies have shown VRE prevalence to vary from $1 \%$ to $9 \%$, which has raised concerns for clinicians to treat difficult enterococcal infections in patients. ${ }^{[14-17]}$ The shift in the antibiotic paradigm has, therefore, made it necessary to investigate the susceptibility pattern of Gram-positive pathogens in order to select an appropriate antibiotic regimen for patients with resistant pathogens. Therefore, the aim of this study is to investigate the prevalence and the antibiotic sensitivity pattern of Gram-positive pathogen in various infections in a tertiary care hospital in Mumbai.

\section{Materials and Methods}

This retrospective cross-sectional study was carried out from January, 2015 to December, 2017, at a tertiary care hospital in Mumbai, India. The patient samples were processed using standard laboratory techniques and were further cultured overnight on Columbia sheep blood agar (CBA) (BioMérieux, France) and on MacConkey's agar (HIMedia, India). After colony characterization and Gram staining, the Gram-positive pathogens were processed and distinguished on the basis of catalase test, slide coagulase test, and tube coagulase test. Further, organism identification and antibiotic susceptibility test was done using the automated VITEK 2 compact system (BioMérieux, France). For streptococci identification, CBA plates were inoculated and Kirby-Bauer disc diffusion testing was done. The antibiotic susceptibility testing was reported as per the Clinical and Laboratory Standards Institute criteria. ${ }^{[18,19]}$

\section{Results}

Prevalence of Gram-positive isolates

A total of 7393 bacterial isolates were analyzed from 2015 to 2017. Of the 2132 Gram-positive pool (29\%), S. aureus $(48.8 \%)$ formed the major encountered pathogen followed by Enterococcus spp. (24.6\%) and coagulase-negative Staphylococcus (CoNS) (15.7\%). Table 1 summarizes the Gram-positive prevalence from 2015 to 2017.

Majority of S. aureus (61.2\%) were isolated from patients with skin and soft-tissue infections (SSTIs) followed by respiratory infections (41.1\%) and BSIs (35.1\%). Out of the total Gram-positive pathogens, $310(14.5 \%)$ were MRSA isolates. Further, the MRSA isolates formed $29.7 \%$ of the total S. aureus identified, whereas the total methicillin-sensitive $S$. aureus (MSSA) prevalence was $67.3 \%$ from 2015 to 2017. Table 2 summarizes the MRSA prevalence from 2015 to 2017. Table 3 summarises system wise infection prevalence of gram positive infections.

Apart from S. aureus, the second most common infection in blood was caused by CoNS pathogens (39.7\%). While $9.2 \%$ of the CoNS species were responsible for infection in patients with SSTIs, no role of these pathogens was observed in respiratory or urinary tract infections. Further, Staphylococcus epidermidis was the most common CoNS species in both BSI and SSTIs, representing $62.4 \%$ and $68 \%$ of the total CoNS species identified, respectively. Staphylococcus haemolyticus represented $12.4 \%$. Other rare CoNS species identified included Staphylococcus hominis (6.5\%), Staphylococcus lugdunensis $(4.5 \%)$, Staphylococcus warneri $(2 \%)$, Staphylococcus xylosus (1.5\%), Staphylococcus capitis (1.2\%),

Table 1: Total prevalence of Gram-positive pathogens in our hospital

\begin{tabular}{lcccc}
\hline $\begin{array}{l}\text { Gram-positive } \\
\text { pathogens }\end{array}$ & $\begin{array}{c}2015 \\
(n=707)\end{array}$ & $\begin{array}{c}2016 \\
(n=711)\end{array}$ & $\begin{array}{c}2017 \\
(n=714)\end{array}$ & $\begin{array}{c}\text { Total prevalence } \\
(\%)\end{array}$ \\
\hline $\begin{array}{l}\text { Staphylococcus } \\
\text { aureus }\end{array}$ & 361 & 338 & 342 & 48.8 \\
Enterococcus spp. & 186 & 175 & 163 & 24.6 \\
CoNS & 81 & 123 & 131 & 15.7 \\
Streptococcus spp. & 76 & 74 & 78 & 10.7 \\
\hline
\end{tabular}

CoNS=Coagulase-negative Staphylococcus

Table 2: Total methicillin-resistant

staphylococcus aureus and methicillin-susceptible staphylococcus aureus prevalence in Gram-positive isolates from 2015 to 2017

\begin{tabular}{l|c|c|c|c}
\hline $\begin{array}{l}\text { Total Gram-positive } \\
\text { isolates }\end{array}$ & $\begin{array}{c}2015 \\
(n=707)\end{array}$ & $\begin{array}{c}2016 \\
(n=711)\end{array}$ & $\begin{array}{c}2017 \\
(n=714)\end{array}$ & $\begin{array}{c}\text { Total prevalence } \\
(\%)\end{array}$ \\
\hline Total S. aureus & 361 & 338 & 342 & 28.8 \\
MRSA & 124 & 81 & 105 & 29.7 \\
MSSA & 223 & 255 & 225 & 67.3 \\
\hline
\end{tabular}

S. aureus=Staphylococcus aureus, MSSA=Methicillin-susceptible $S$. aureus, MRSA=Methicillin-resistant $S$. aureus 
Table 3: Total prevalence of Staphylococcus aureus, coagulase-negative Staphylococcus, Streptococcus spp., and Enterococcus spp.

\begin{tabular}{ll|c|c|c|c}
\hline Pathogen & Infection & 2015 & 2016 & 2017 & $\begin{array}{c}\text { Total } \\
\text { prevalence (\%) }\end{array}$ \\
\hline S. aureus & BSI $(n=435)$ & 83 & 44 & 26 & $153(35.10)$ \\
& SSTIs $(n=1355)$ & 262 & 268 & 300 & $830(61.2)$ \\
CoNS & RTI $(n=68)$ & 3 & 16 & 9 & $28(41.1)$ \\
& BSI $(n=435)$ & 51 & 64 & 58 & $173(39.7)$ \\
Streptococcus & SSTIs $(n=1355)$ & 21 & 43 & 61 & $125(9.2)$ \\
spp. & BSI $(n=435)$ & 26 & 20 & 10 & $56(12.8)$ \\
& SSTIs $(n=1355)$ & 35 & 50 & 45 & $130(9.6)$ \\
Enterococcus & RTI $(n=68)$ & 13 & 3 & 16 & $32(47)$ \\
spp. & BSI $(n=435)$ & 14 & 22 & 15 & $51(11.7)$ \\
& SSTIs $(n=1355)$ & 93 & 88 & 86 & $267(19.7)$ \\
\hline CoNS Coal $(n=175)$ & 69 & 55 & 43 & $167(95.4)$ \\
\hline
\end{tabular}

CoNS=Coagulase-negative Staphylococcus, BSI=Bloodstream infection, SSTIs=Skin and soft-tissue infections, RTI=Respiratory tract infection, S. aureus=Staphylococcus aureus

Staphylococcus urealyticus $(<1 \%)$, Staphylococcus sciuri $(<1 \%)$, and Staphylococcus saprophyticus $(<1 \%)$.

Streptococcus spp. were the most dominant pathogen isolated in patients with respiratory tract infection (RTI) (47\%), whereas in BSI and SSTIs, it was $12.9 \%$ and $9.6 \%$, respectively. While Streptococcus pneumoniae (37.5\%) was the most common Streptococcus spp. in RTI patients, patients with BSI and SSTIs also had Streptococcus pyogenes (12.2\%), Streptococcus agalactiae (10\%), Streptococcus mitis (1.8\%), Streptococcus dys equisimilis $(<1 \%)$, Streptococcus anginosus $(<1 \%)$, and Streptococcus pasteurianus $(<1 \%)$.

On the other hand, the total prevalence of Enterococcus faecalis was $30.8 \%$ while that of E. faecium was $8.4 \%$ of the total isolated Enterococcus species. In addition, Enterococcus spp. were the major causating pathogen behind urinary tract infections (95.4\%), whereas only $19.7 \%$ and $11.7 \%$ were responsible for SSTIs and BSI, respectively.

Antibiotic sensitivity pattern in Staphylococcus aureus and Enterococcus spp.

An antibiotic sensitivity pattern for ciprofloxacin, erythromycin, clindamycin, co-trimoxazole, linezolid, vancomycin, daptomycin, tigecycline, and teicoplanin was determined. MRSA isolates showed lower sensitivity toward ciprofloxacin $(10.2 \%)$, erythromycin $(24 \%)$, clindamycin $(30.2 \%)$, and co-trimoxazole $(39.2 \%)$ as compared to MSSA isolates [Figure 1].

Further, MRSA was shown to be highly susceptible toward linezolid (98\%), vancomycin $(97.7 \%)$, and teicoplanin (98.3\%). All MRSA and MSSA isolates tested were found to be sensitive to daptomycin and tigecycline $(100 \%)$. Moreover, none of the isolates were found to be sensitive to benzyl penicillin.

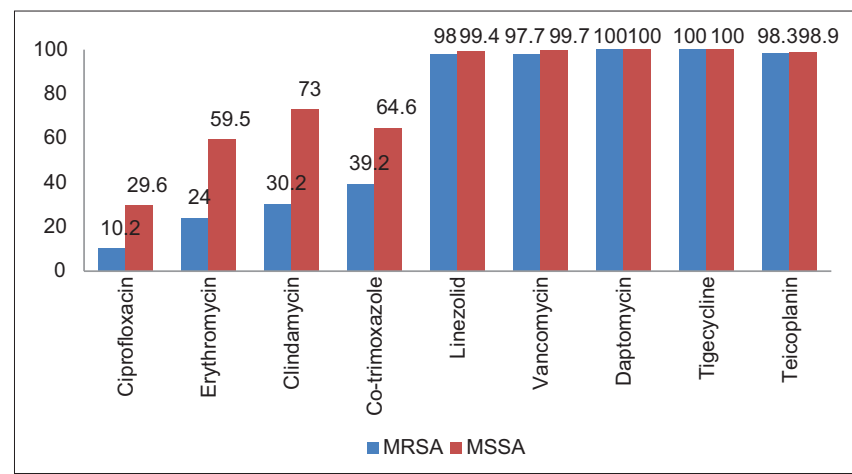

Figure 1: Antibiotic sensitivity pattern in methicillin-resistant Staphylococcus aureus and methicillin-sensitive Staphylococcus aureus isolates

We also investigated the sensitivity pattern in Enterococcus spp., where they were highly susceptible to linezolid (96\%), vancomycin (92\%), and teicoplanin (93.3\%) while being resistant to erythromycin and ciprofloxacin. Further, the prevalence of VRE was found to be $7.5 \%$. Figure 2 shows the sensitivity pattern for Enterococcus spp.

\section{Discussion}

MDR bacterial infections have today become the biggest threat in the world due to the irrational use of antimicrobial agents. The European Prevalence of Infection in Intensive Care 2 study showed that, out of $47 \%$ of the Gram-positive isolates, $20 \%$ were S. aureus which was further associated with longer ICU stay. ${ }^{[20]}$ In India too, Gram-positive infections, particularly MRSA, have been reported to increase exponentially from $29 \%$ in 2009 to $47 \%$ in 2014. ${ }^{[21]}$ The overall MRSA prevalence in our study was $29.7 \%$, which was lower than the recently reported prevalence of $37.3 \%$ by ICMR-AMRSN ${ }^{[8]}$ and $40 \%$ as reported by INSAR study. ${ }^{[22]}$ Furthermore, the prevalence varies among different centers across India ranging from $20.2 \%$ in Gujarat to $80.4 \%$ in Odisha. This variation could be attributed to the fact that different centers have different patient profiles and local antibiotic regimens that could influence the overall prevalence.

The ICMR-AMRSN study reported $S$. aureus to be the most frequently isolated organism in SSTI patients $(73.7 \%))^{[8,23]}$ Similarly, our study also showed similar results with S. aureus isolates to be $61.2 \%$ in SSTI patients. Further, we also report a high prevalence of $S$. aureus $(35.1 \%)$ in BSI patients. Recently, apart from SSTIs, $S$. aureus has also emerged to be a critical pathogen in BSIs. ${ }^{[24]}$ This is further supported by a study conducted by Tak et al. where the crude mortality rate in patients with MRSA BSI was 31\%. ${ }^{[25]}$

In 2010, India was the highest antibiotic consumer, with 10.7 units being consumed per person. Among BRICS countries, India registered an increase of $23 \%$ in the retail sale volume. ${ }^{[26]}$ Therefore, easy availability 


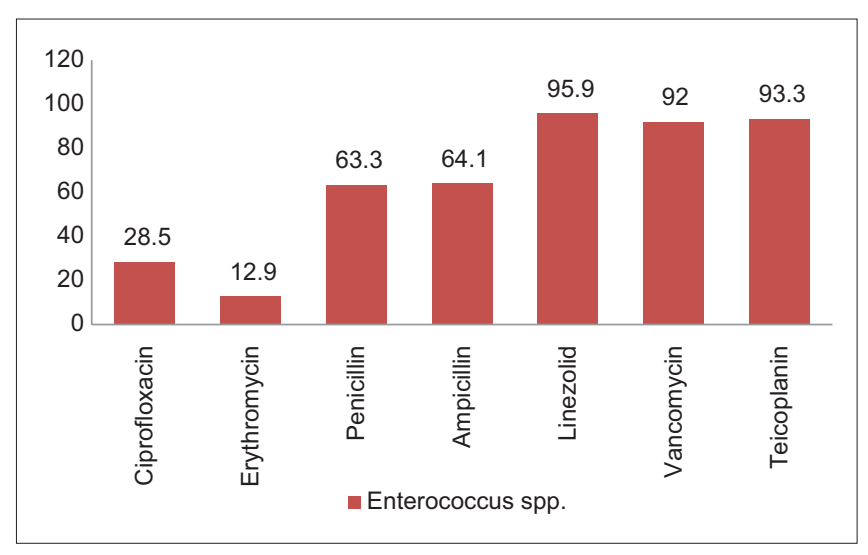

Figure 2: Antibiotic sensitivity pattern of Enterococcus species

and inappropriate antibiotic abuse has resulted in the development of antimicrobial resistance, which is difficult to treat and has become a national health disaster, today. In the present study, the MRSA isolates showed higher resistance toward ciprofloxacin, erythromycin, co-trimoxazole, and clindamycin as compared to MSSA isolates. Similar observations were also seen in previous multicentric studies. ${ }^{[8,22]}$ These findings justify the increased mortality rates ${ }^{[27]}$ among patients infected with MRSA infections due to the ability of the pathogen to display varied resistance patterns toward various antibiotics. Moreover, both daptomycin and tigecycline remained the most effective antibiotics against $S$. aureus because there was no resistant isolate observed. This effect could be attributed to two reasons, with the higher cost of the drugs to be the primary reason due to which these drugs are not used very commonly. Second, both tigecycline and daptomycin are not the antibiotic of choice for treating BSIs and lung infections, both of which are the most common sources of infection in our hospital.

Vancomycin has been regarded as the first-line drug for the treatment of MRSA and MDR Enterococcus spp. Vancomycin was highly effective against MRSA isolates as we documented $<1 \%$ of vancomycin-intermediate strains in them. However, a meta-analysis has shown vancomycin-intermediate $S$. aureus (VISA) and hetero-VISA prevalence to be $15.09 \%$ and $12.41 \%$, respectively. ${ }^{[28]}$ While we observed only three $(<1 \%)$ vancomycin-resistant isolates, a study by Singh et al. has reported $13 \%$ of VRSA isolates. ${ }^{[29]}$

The first vancomycin-resistant E. faecalis and E. faecium was reported in 1988 in England. ${ }^{[30]}$ Subsequently, the first case of VRE was reported from New Delhi in $1999^{[31]}$ because of unscrupulous use of vancomycin for Enterococcus treatment. In India, the prevalence of VRE infection is increasing with every passing year. In 2003, the VRE prevalence was $1 \%{ }^{[14]}$ which in 2013 has risen up to $8.7 \% .^{[17]}$ In the present study too, a total of $39(7.5 \%)$ VRE isolates were obtained. Moreover, eight isolates (1.5\%) of E. gallinarum and E. casseliflavus, which have inherent resistance to vancomycin, were also observed. However, out of the eight isolates, one isolate was vancomycin, linezolid, and teicoplanin resistant.

Linezolid is the only oral antibiotic available as compared to its other counterparts for the treatment of resistant staphylococcus species. Furthermore, linezolid is used as a reserved drug for the treatment of difficult extremely drug-resistant tuberculosis and extensively drug-resistant tuberculosis. ${ }^{[32]}$ However, linezolid overuse in the management of Gram-positive pathogens, which are otherwise easy to treat, has caused an emergence of linezolid-resistant strains. Globally, there are not much reports about the prevalence of linezolid-resistant S. aureus $(<1 \%)$. A study from Rajasthan showed $12 \%$ of the linezolid-resistant $S$. aureus; ${ }^{[33]}$ however, we show that $98 \%$ of the MRSA isolates were sensitive to linezolid, making it a potent drug for activity against resistant staphylococcus organisms. Further, in case of Enterococcus, linezolid is a successful therapeutic option if vancomycin treatment fails. Kumar et al. first reported linezolid-resistant enterococci (LRE) in 2014. ${ }^{[34]}$ However, there are no further reports which document the prevalence of LRE, thereby making this the first study to show a high LRE prevalence of $4.1 \%$. The high prevalence of LRE in our hospital may have resulted due to the increased MRSA prevalence which further corresponds to an increase in the use of linezolid empirically. Due to this, the antimicrobial stewardship program in the hospital has taken measures and has tightened the program in order to keep a check on the increasing resistant strains.

\section{Conclusion}

This study demonstrates MRSA and VRE as a growing health problem in India. While, vancomycin and linezolid remain the preferred choice for MRSA and multiresistant Enterococcus treatment, the rise in both vancomycin- and linezolid-resistant strains has caused treatment dilemmas for clinicians. Therefore, the data generated provide in-depth knowledge of the changing antibiotic regimen against the difficult pathogens, thereby helping the physicians to make the right antibiotic choice at the start of the treatment. Further, there is an urgent need for new research molecules to combat the difficult MDR pathogens.

\section{Acknowledgment}

We thank Dr. Ritika Rampal, Dr. Jaishid Ahdal, and Wockhardt Ltd, an Indian pharmaceutical company for writing the manuscript.

\section{Financial support and sponsorship} Nil. 


\section{Conflicts of interest}

There are no conflicts of interest.

\section{References}

1. Lozano R, Naghavi M, Foreman K, Lim S, Shibuya K, Aboyans V, et al. Global and regional mortality from 235 causes of death for 20 age groups in 1990 and 2010: A systematic analysis for the global burden of disease study 2010. Lancet 2012;380:2095-128.

2. Murray CJ, Vos T, Lozano R, Naghavi M, Flaxman AD, Michaud C, et al. Disability-adjusted life years (DALYs) for 291 diseases and injuries in 21 regions, 1990-2010: A systematic analysis for the global burden of disease study 2010. Lancet 2012;380:2197-223.

3. John TJ, Dandona L, Sharma VP, Kakkar M. Continuing challenge of infectious diseases in India. Lancet 2011;377:252-69.

4. Laxminarayan R, Chaudhury RR. Antibiotic resistance in India: Drivers and opportunities for action. PLoS Med 2016;13:e1001974.

5. Mave V, Chandanwale A, Kagal A, Khadse S, Kadam D, Bharadwaj R, et al. High burden of antimicrobial resistance and mortality among adults and children with community-onset bacterial infections in India. J Infect Dis 2017;215:1312-20.

6. Tong SY, Davis JS, Eichenberger E, Holland TL, Fowler VG Jr. Staphylococcus aureus infections: Epidemiology, pathophysiology, clinical manifestations, and management. Clin Microbiol Rev 2015;28:603-61.

7. National Treatment Guidelines for Antimicrobial Use in Infectious Diseases. India: National centre for disease control, Directorate General of Health Services, Ministry of Health and family welfare, Government of India; 2016 (version 1). p. 2.

8. RajkumarS, SistlaS, Manoharan M, Sugumar M,Nagasundaram N, Parija SC, et al. Prevalence and genetic mechanisms of antimicrobial resistance in Staphylococcus species: A multicentre report of the Indian council of medical research antimicrobial resistance surveillance network. Indian J Med Microbiol 2017;35:53-60.

9. Trivedi MB, Vegad M, Soni S. Prevalence of methicillin-resistant Staphylococcus aureus in various clinical samples in a tertiary-care hospital. Int J Med Sci Public Health 2015;4:1735-8.

10. Jindal N, Malhotra R, Grover P, Singh S, Bansal R, Kaur S, et al. Methicillin resistant Staphylococcus aureus (MRSA) in Malwa region of Punjab (North-west India). Indian J Med Res 2016;143:371-2.

11. Gupta R, Malik A, Rizvi M, Ahmed M, Hashmi A. Multidrug resistant Gram positive pathogens with special reference to MRSA and biofilm production in ICU patients: Recurrent challenge for clinicians. Int J Curr Microbiol App Sci 2015;1:207-12.

12. Dubey D, Rath S, Sahu MC, Pattnaik L, Debata NK, Padhy RN. Surveillance of infection status of drug resistant Staphylococcus aureus in an Indian teaching hospital. Asian Pac J Trop Dis 2013;3:133-42.

13. Sood S, Malhotra M, Das BK, Kapil A. Enterococcal infections \& amp; antimicrobial resistance. Indian J Med Res 2008;128:111-21.

14. Mathur P, Kapil A, Chandra R, Sharma P, Das B. Antimicrobial resistance in Enterococcus faecalis at a tertiary care centre of Northern India. Indian J Med Res 2003;118:25-8.

15. Taneja N, Rani P, Emmanuel R, Sharma M. Significance of vancomycin resistant enterococci from urinary specimens at a tertiary care centre in Northern India. Indian J Med Res 2004;119:72-4.

16. Kaur N, Chaudhary U, Aggarwal R, Bala K. Emergence of VRE and their antimicrobial sensitivity pattern in a tertiary care teaching hospital. J Med Biol Sci 2009;8:26-32.

17. Praharaj I, Sujatha S, Parija SC. Phenotypic \& genotypic characterization of vancomycin resistant Enterococcus isolates from clinical specimens. Indian J Med Res 2013;138:549-56.

18. Clinical and Laboratory Standards Institute. Performance Standards for Antimicrobial Susceptibility Testing. $25^{\text {th }}$ ed. CLSI Supplement M100. Wayne, PA: Clinical and Laboratory Standards Institute; 2015.

19. Clinical and Laboratory Standards Institute; Performance Standards for Antimicrobial Susceptibility Testing. $26^{\text {th }}$ ed. CLSI Supplement M100S. Wayne, PA: Clinical and Laboratory Standards Institute; 2016.

20. Vincent JL, Rello J, Marshall J, Silva E, Anzueto A, Martin CD, et al. International study of the prevalence and outcomes of infection in intensive care units. JAMA 2009;302:2323-9.

21. Center for Disease Dynamics, Economics \& Policy. Resistance Map. Washington DC: Center for Disease Dynamics, Economics \& Policy; 2015.

22. Indian Network for Surveillance of Antimicrobial Resistance (INSAR) group, India. Methicillin resistant Staphylococcus aureus (MRSA) in India: Prevalence \& susceptibility pattern. Indian J Med Res 2013;137:363-9.

23. Saseedharan S, Sahu M, Chaddha R, Pathrose E, Bal A, Bhalekar P, et al. Epidemiology of diabetic foot infections in a reference tertiary hospital in India. Braz J Microbiol 2018;49:401-6.

24. Patel AK, Patel KK, Patel KR, Shah S, Dileep P. Time trends in the epidemiology of microbial infections at a tertiary care center in west India over last 5 years. J Assoc Physicians India 2010;58 (Suppl):37-40.

25. Tak V, Mathur P, Lalwani S, Misra MC. Staphylococcal blood stream infections: Epidemiology, resistance pattern and outcome at a level 1 Indian trauma care center. J Lab Physicians 2013;5:46-50.

26. Van Boeckel TP, Gandra S, Ashok A, Caudron Q, Grenfell BT, Levin SA, et al. Global antibiotic consumption 2000 to 2010: An analysis of national pharmaceutical sales data. Lancet Infect Dis 2014;14:742-50.

27. Gandra S, Tseng KK, Arora A, Bhowmik B, Robinson ML, Panigrahi B, et al. The mortality burden of multidrug-resistant pathogens in India: A retrospective observational study. Clinical Infectious Diseases 2018.

28. Zhang S, Sun X, Chang W, Dai Y, Ma X. Systematic review and meta-analysis of the epidemiology of vancomycin-intermediate and heterogeneous vancomycin-intermediate Staphylococcus aureus isolates. PLoS One 2015;10:e0136082.

29. Singh H, Atray M, Modi PK. Antibiotic susceptibility pattern of methicillin resistance Staphylococcus aureus in tertiary care center at southern Rajasthan. Int J Pharm Sci Res 2014;5:607-11.

30. Uttley AH, Collins CH, Naidoo J, George RC. Vancomycin-resistant enterococci. Lancet 1988;1:57-8.

31. Mathur P, Chaudhary R, Dhawan B, Sharma N, Kumar L. Vancomycin-resistant Enterococcus bacteraemia in a lymphoma patient. Indian J Med Microbiol 1999;17:194-5.

32. Maartens G, Benson CA. Linezolid for treating tuberculosis: A delicate balancing act. EBioMedicine 2015;2:1568-9.

33. Pavani G. Linezolid resistant Staphylococcus aureus. Int J Res Med Sci 2017;2:1253-6.

34. Kumar S, Bandyoapdhyay M, Chatterjee M, Mukhopadhyay P, Poddar S, Banerjee P, et al. The first linezolid-resistant Enterococcus faecium in India: High level resistance in a patient with no previous antibiotic exposure. Avicenna J Med 2014;4:13-6. 